\title{
Meta
}

Journal des traducteurs

Translators' Journal

\section{ORdóñez Ló Pez, Pilar y SABio Pinilla, JosÉ ANTONio (2015): Historiografía de la traducción en el espacio ibérico. Textos contemporáneos. Cuenca: Ediciones de la Universidad de Castilla-La Mancha, 387 p.}

\section{Aura E. Navarro}

Volume 62, numéro 2, août 2017

URI : https://id.erudit.org/iderudit/1041034ar

DOI : https://doi.org/10.7202/1041034ar

Aller au sommaire du numéro

Éditeur(s)

Les Presses de l’Université de Montréal

ISSN

0026-0452 (imprimé)

1492-1421 (numérique)

Découvrir la revue

Citer ce compte rendu

Navarro, A. E. (2017). Compte rendu de [ORDóÑEZ LÓPEZ, PILAR Y SABIO PINILlA, JosÉ ANTONIO (2015): Historiografía de la traducción en el espacio ibérico. Textos contemporáneos. Cuenca: Ediciones de la Universidad de Castilla-La Mancha, 387 p.] Meta, 62(2), 464-467.

https://doi.org/10.7202/1041034ar d'utilisation que vous pouvez consulter en ligne. 
partage des compétences entre les spécialistes de la traduction et de la langue, et les spécialistes du droit ou d'autres domaines dans la production des textes traduits et la normalisation des termes en usage dans ces domaines de spécialisation.

Éric PoIrIER Université du Québec à Trois-Rivières, Trois-Rivières, Canada

\section{NOTES}

1. La comparaison avec certains de ces ouvrages peut surprendre. Puisque certains de ces ouvrages relèvent d'une spécialisation différente ou visent une autre langue source, la comparaison ne constitue en aucune façon une évaluation de leur contenu, mais bien pour ce qui concerne le choix des entrées, des termes ou des articles à intégrer ou non dans la nomenclature.

2. On en a relevé quelques-unes marquées par CAN, surtout pour les notions de common law particulièrement difficiles à traduire comme fee simple ou fee tail, mais aussi lorsque l'emprunt est suggéré en français européen, comme c'est le cas pour la traduction de forum shopping.

\section{RÉFÉRENCES}

GARner, Bryan A. (dir.) (2004). Black's Law Dictionary. Eighth Edition. Saint Paul, Minn.: Thomson/West.

GÉmar, Jean-Claude et Ho-Thuy, Vo (2016). Nouvelles difficultés du langage du droit - Dits et maux de Thémis. Montréal: Éditions Thémis.

Houbert, Frédéric (2000). Dictionnaire des difficultés de l'anglais des contrats. Paris: Maison du Dictionnaire.

Houbert, Frédéric (2005). Guide pratique de la traduction juridique: anglais-français. Paris: Maison du Dictionnaire.

Lapointe-Giguère, Micheline et Office QuÉBÉCOIS DE LA LANGUE FRANÇAISE (2009). Vocabulaire des relations professionnelles français-anglais. Montréal: Presses internationales Polytechnique.

MénARD, Louis (2004). Dictionnaire de la comptabilité et de la gestion financière anglais-français avec index français-anglais. Deuxième édition. Toronto: Institut canadien des comptables agréés.

PoIrIER, Éric (2009). «Une référence indispensable pour langagiers débutants et chevronnés.» Circuit. 105. Montréal: Ordre des traducteurs, terminologues et interprètes agréés du Québec. Compte rendu du Vocabulaire des relations professionnelles français-anglais (2009) de Micheline Lapointe-Giguère et OFFICE QUÉBÉCOIS DE LA LANGUE FRANÇAISE. En ligne: https://ottiaq.org/upload/circuit/ CI_106_10.pdf

Poirier, Éric (2010). «Quand la technologie se met au service d'une véritable bidirectionnalité...»
Circuit. 106. Montréal: Ordre des traducteurs, terminologues et interprètes agréés du Québec. Compte rendu du Dictionnaire des dérivés et autres instruments financiers (2009) de Louis Ménard et Antoni Dandonneau. En ligne: http://publidict.ocaq.qc.ca

Poirier, Éric (2015). «La voie électronique vers la valeur saussuréenne des termes des domaines de l'économie.» Circuit. 126. Montréal: Ordre des traducteurs, terminologues et interprètes agréés du Québec. Compte rendu du Dictionnaire analytique de la mondialisation et $d u$ travail DAMT (2013) de Jeanne DANCETTE. En ligne: www.circuitmagazine.org/archives

ReID, Hubert (2004). Dictionnaire de droit québécois et canadien. Troisième édition. Montréal: Wilson et Lafleur.

Ordóñez López, Pilar y Sabio Pinilla, José Antonio (2015): Historiografía de la traducción en el espacio ibérico. Textos contemporáneos. Cuenca: Ediciones de la Universidad de CastillaLa Mancha, 387 p.

Historiografía de la traducción en el espacio ibérico es la decimosexta obra de la colección titulada "Escuela de Traductores de Toledo", dirigida por el centro de enseñanza e investigación que lleva el mismo nombre. Este centro se especializa en formar traductores del árabe y del hebreo al español. Algunos de los títulos publicados en la colección incluyen: Traducir al Otro: traducción, exotismo, poscolonialismo (Carbonell i Cortés, 1997), Teorías de la Traducción: Antología de textos (López García, 1996) y La traducción periodística (Cortés Zaborras y Hernández Guerrero, 2005).

El proyecto inicial de los editores de Historiografía de la traducción en el espacio ibérico, Pilar Ordóñez López y José Antonio Sabio Pinilla, era la publicación de una antología (reader) sobre historiografía de la traducción. El reader incluiría textos publicados durante los últimos cuarenta años que reflejaran una visión amplia, no solo eurocéntrica. La idea se transformó en una obra más delimitada, la cual recoge textos en castellano, portugués y catalán, representativos del trabajo de investigación que ha tenido lugar en el espacio ibérico en las últimas dos décadas. En este sentido, tal como lo sugieren sus editores, la obra es el complemento de Las antologías sobre la traducción en el ámbito peninsular. Análisis y estudio, donde se analizan quince antologías publicadas en el ámbito peninsular entre 1987 y 2011 (véase Sabio Pinilla y Ordóñez López, 2012).

Ordóñez López y Sabio Pinilla tienen una larga trayectoria en el campo de la traducción. Ambos son profesores en departamentos de traducción, en la Universitat Jaume I y en la Univer- 
sidad de Granada, respectivamente. Han publicado además numerosos trabajos relacionados con la historia de la traducción y los estudios de traducción.

En Historiografía de la traducción en el espacio ibérico, los editores se plantearon como objetivo "mostrar la singularidad de la investigación histórica en traducción de la Península Ibérica señalando la pujanza de su estudio y la riqueza de los temas tratados." (p. 10) Los editores aclaran que, dejando de lado las connotaciones políticas que el término "ibérico" pueda tener, en esta obra se emplea como una "categoría supranacional" que les permite "encuadrar la historia de un conjunto de sistemas lingüísticos y literarios dentro de un espacio cultural común." (ibid.) El libro es una clara apuesta por lo "local" que busca hacer un acercamiento historiográfico enfocado en el ámbito ibérico, aunque no de una manera aislada o inconexa, es decir, sin dejar de lado la perspectiva "global".

Reunieron pues quince textos y diecinueve autores de diferentes horizontes. Todos ellos expresan una misma preocupación, la de "describir, analizar y orientar la investigación histórica.” (p. 12) Historiografía de la traducción en el espacio ibérico está dividida en tres bloques temáticos que, lejos de ser "compartimientos estancos", sirven de guía e ilustran los grandes temas y enfoques que se pueden encontrar en la historiografía ibérica. Cada bloque está acompañado de una introducción muy pertinente que no se limita a presentar los textos, sino que contextualiza muy acertadamente la temática tratada en cada una de las partes.

El primer bloque temático, titulado "Tradición filológica, literaria y comparada”, comprende cuatro textos que presentan una investigación enfocada en la literatura traducida y en su impacto en los diferentes sistemas literarios en el ámbito ibérico. En el primer artículo, Miguel Gallego Roca (1994) estudia el rol de las traducciones en la historia literaria nacional y propone la teoría del polisistema como marco adecuado para el estudio de las traducciones literarias. Gallego Roca expresa, además, la necesidad de escribir una historia de la traducción en España, un deseo ahora cumplido. El texto de José Francisco Ruiz Casanova (2000), que es la nota preliminar de su libro Aproximación a una Historia de la Traducción en España, es un estudio pionero donde se presenta un panorama de la historia de la traducción de España como un conjunto. Ruiz Casanova, que propone una periodización que va desde la Edad Media hasta el siglo XX, resalta cómo la traducción va de la mano de la lengua y la literatura en una comunidad. Por su parte, el artículo de Salvador Peña Martín (2005) aborda la historia de la traducción del árabe al castellano, resaltando el aporte del primero al segundo. Su trabajo sobre los traductores y los agentes de traducción muestra la utilidad del modelo narrativo en el estudio de la historia de la traducción. El texto de Julio César Santoyo (2010), el cual concluye este primer bloque, aborda el fenómeno de la autotraducción en la península ibérica, tomando en cuenta las circunstancias histórico-personales de los autores-traductores.

El segundo bloque se titula "Enfoques historiográficos propiamente dichos" y recoge cinco textos que tratan de cuestiones metodológicas. En general, estos trabajos resaltan la importancia de adoptar una perspectiva interdisciplinar en la investigación histórica. El primer texto es de Brigitte Lépinette (1997) con su propuesta metodológica para el estudio de la historia de la traducción. Este trabajo pionero, en el cual Lépinette propone dos modelos fundamentales (sociológico-cultural e histórico-descriptivo), ha tenido una gran influencia en la investigación historiográfica de la traducción y ha sido, desde entonces, abundantemente citado. El trabajo de Ma Manuela Fernández Sánchez y José Antonio Sabio Pinilla (2003) cuestiona los métodos historiográficos de la traducción empleados en el ámbito ibérico, lo que ha provocado una historia repetitiva, estancada y aislada, según los autores. Ellos abogan por un relato histórico con capacidad narrativa, por un tratamiento adecuado de las fuentes y sus contextos y por la selección del material según ciertos criterios de coherencia, para la interpretación de los significados culturales, sociales y culturales. Icíar Alonso (2008), por su parte, se enfoca en la historia de la interpretación, específicamente en el estudio de las fuentes, área poco explorada hasta ahora. Miguel Ángel Vega y Martha Pulido (2013) estudian la trayectoria de los Estudios de Traducción como disciplina y resaltan el lugar que debe ocupar la historia de la traducción dentro de un programa universitario. Subrayan los autores la complejidad que representa establecer una periodización propia a la disciplina, y apuntan a que se debe seguir escribiendo la historia de la traducción, resaltando sobre todo el papel del traductor y el rol de las traducciones en el progreso de las sociedades.

Vega y Pulido concluyen su trabajo con un apéndice bastante escueto sobre la historia de la traducción en Hispanoamérica. Si bien es cierto que Vega y Pulido lo califican de "brevísimo recorrido" de la investigación que se está haciendo en América Latina y sobre América Latina, no se justifica que en este apéndice solo mencionen unos seis autores y que se deje de lado el trabajo que otros investigadores han venido haciendo desde hace unas dos décadas - por ejemplo, en el grupo de investigación HISTAL (Historia de la traducción en América Latina) de la Universidad 
de Montreal. El comentario de unas cuatro líneas que los editores del libro agregan en la nota introductoria de este segundo bloque o una mención efímera en una nota al pie por los autores Vega y Pulido, no dan cuenta de la labor que se ha estado haciendo en el grupo HISTAL. Además de numerosas publicaciones por parte de Georges L. Bastin y de los otros integrantes de este grupo de investigación ${ }^{1}$, el sitio web de HISTAL (www.histal. ca) cuenta con un reconocimiento internacional que ha permitido establecer contactos valiosos entre investigadores de alto calibre. Por otra parte, al hablar de la necesidad de una periodización de la historia de la traducción en América Latina, Vega y Pulido no mencionan la periodización ${ }^{2}$ propuesta por Bastin a finales de los noventa en su texto Latin American Tradition, publicado en la Routledge Encyclopedia of Translation Studies (Bastin, 1998). Aquí vienen al caso las palabras de Fernández Sánchez y Sabio Pinilla cuando, haciendo referencia a la investigación histórica de la traducción en el ámbito ibérico, dicen: "Seguimos sin leernos, sin intercambiar informaciones [...].” (p. 167) Tristemente esto se aplica al texto de Vega y Pulido. Para seguir escribiendo la historia de la traducción en Hispanoamérica, debemos empezar por "leernos", “intercambiar informaciones” y agregaría, dar crédito al trabajo valioso que ya se ha hecho. Terminaré este comentario señalando que, como lo comentan Vega y Pulido, todavía hay mucho trabajo por hacer en lo que respecta a la historia de la traducción en Hispanoamérica. Al respecto se hace imperativo mencionar el aporte valiosísimo del Diccionario histórico de la traducción en Hispanoamérica (Lafarga y Pegenaute, 2013), el cual se publica un poco antes del libro reseñado en el presente texto. Este Diccionario reúne a más de cien investigadores y que ofrece una "visión de conjunto" sobre la historia de la traducción en la Región.

Pero retomemos ahora el libro de Ordóñez López y Sabio Pinilla. El último texto del segundo bloque, cuya autora es Teresa Seruya (2013), aborda la importancia de la elaboración de una historia de la traducción en Portugal, tomando en cuenta no solo la literatura traducida sino la censurada. El proyecto de investigación que ella dirige aborda la traducción de la literatura publicada en Portugal de 1930 a 2000.

El tercer bloque lleva por título "Enfoques ideológicos y poscoloniales” y está conformado por seis artículos que, en términos generales, tratan de la relación con el Otro, la mediación lingüística en situaciones de conflicto y la relación asimétrica entre lenguas y culturas. Ovidi Carbonell (1997, 1999) abre este bloque con su estudio sobre la traducción desde una perspectiva postcolonial. Según el autor, en contextos de litigio, la traducción puede no solo emplearse como una herramienta para escribir la historia desde la perspectiva del colonizador, sino también como un medio de subversión para luchar contra las representaciones dominantes. Por su parte, Nora Catelli y Marietta Gargatagli (1998) le dan una voz a los "excluidos" de la Historia, en el contexto de conquista y colonización en Hispanoamérica. Este texto es el prólogo de la antología El tabaco que fumaba Plinio (Catelli y Gargatagli, 1998). Francesc Parcerisas (2000) estudia cómo la traducción puede dejar ver la relación asimétrica entre diferentes lenguas. Tomando como ejemplo una lengua minoritaria en el contexto ibérico como lo es la catalana, Parcerisas recalca el doble rol de la traducción del catalán hacia otras lenguas (como el inglés o el español), ya que refleja la situación de asimetría existente, al mismo tiempo que permite una mayor divulgación a una lengua minoritaria, la catalana. Gertrudis Payàs Puigarnau (2010) aborda la historia de los intérpretes en el contexto chileno del siglo XVII, especialmente en lo que respecta a las relaciones de alteridad en este contexto colonial. Jesús Baigorri Jalón (2012) se enfoca igualmente en la historia de los intérpretes, aunque su objeto de estudio son los intérpretes que participaron en la guerra civil española. Baigorri resalta la dificultad de establecer un perfil exacto de los intérpretes de guerra, dado que muchos aprendieron este oficio de manera improvisada, obligados por la situación. El texto de Juan Pablo Arias Torres y Manuel C. Feria García (2012) cierra este bloque y aborda la historia de los traductores e intérpretes del Estado español que formaron parte del Cuerpo de Interpretación del Árabe y Bereber, desde principios del siglo XX hasta principios del siglo XXI. Llaman la atención la novedad y diversidad de las fuentes empleadas por los autores, entre las cuales se encuentran la entrevista y las fotografías.

Historiografía de la traducción en el espacio ibérico representa sin duda un aporte importante para este campo de estudio. Una de las grandes riquezas de este libro es que los trabajos aquí recopilados manifiestan una concepción bastante amplia de la historiografía. Por un lado, el acercamiento a la historiografía se hace desde diferentes áreas de estudio (la filología, la literatura comparada y los estudios postcoloniales, por mencionar algunas). Por otro lado, los textos tratan cuestiones bastante diversas que incluyen planteamientos teóricos, metodológicos e ideológicos. Además, la presentación cronológica de los textos en cada bloque es bastante conveniente, ya que permite apreciar cómo la historiografía de la traducción en el ámbito ibérico ha ido evolucionando en los últimos años.

Así, pues, es notorio el avance a pasos agigantados de la historiografía de la traducción en 
el espacio ibérico. España, por su parte, es bien conocida por las numerosas publicaciones en la disciplina, por lo que en la actualidad se cuenta con una historia de la traducción española bastante completa. Destacan varias obras de referencia. En primer lugar, las dirigidas por Francisco Lafarga y Luis Pegenaute: Historia de la traducción en España y Diccionario histórico de la traducción en España (Lafarga y Pegenaute, 2004; 2009, respectivamente). Luego la de Ruiz Casanova, ya mencionada, y la de Santoyo (2009): La traducción medieval en la Península ibérica (siglos III-XV). El caso de Portugal es diferente, pues no existe una obra que reseñe la historia de la traducción como un conjunto. Existen, sin embargo, trabajos que abordan una época en específico (por ejemplo, Castilho Pais, 2003 y Sabio Pinilla, 2009). Aplaudimos la iniciativa de Ordóñez López y Sabio Pinilla en su Historiografía de la traducción en el espacio ibérico, pues la obra sigue enriqueciendo la historiografía de la traducción en la península ibérica.

Aura E. Navarro Grupo de investigación HISTAL (Historia de la traducción en América Latina) Université de Montréal, Montréal, Canada

\section{NOTAS}

1. Véase la sección Documentos en el sitio web del grupo HISTAL (http://www.histal.ca/es/documents/).

2. En su artículo Translation, History and the Translation Scholar, Clara Foz (2006) discute el tema de la periodización en historia de la traducción.

\section{REFERENCIAS}

BAstin, Georges (1998). Latin American Tradition (Traduit par M. Gregson). En: Mona BAKER \& Kirsten MalmkjÆr (dir.), Routledge Encyclopedia of Translation Studies, London: Routledge, 505-512.

Carbonell i Cortés, Ovidi (1997). Traducir al otro: traducción, exotismo, poscolonialismo. Cuenca: Ediciones de la Universidad de Castilla-La Mancha.

Castilho Pais, Carlos (2003). Aspectos de la traducción oral en Portugal en el siglo XVI. En: José A. Sabio Pinilla y $\mathrm{M}^{\mathrm{a}}$ Dolores Valencia (dir.), Seis estudios sobre la traducción en los siglos XVI y XVII. Granada: Comares, 169-204.

Catelli, Nora \& Gargatagli, Marietta (1998). El tabaco que fumaba Plinio: escenas de la traducción en España y América: relatos, leyes y reflexiones sobre los otros. Barcelona: Del Serbal.

Cortés Zaborras, Carmen \& Hernández GueRRERO, Ma José (dir.). (2005). La traducción periodística. Cuenca: Ediciones de la Universidad de Castilla-La Mancha.
Foz, Clara (2006). Translation, History and the Translation Scholar. En: Georges L. Bastin \& Paul Bandia (dirs.). Charting the Future of Translation History. Ottawa: University of Ottawa Press, 131-144.

Lafarga, Francisco \& Pegenaute, Luis (2004). Historia de la traducción en España. Ambos Mundos: Salamanca.

Lafarga, Francisco \& Pegenaute, Luis (2009). Diccionario histórico de la traducción en España. Gredos: Madrid.

López García, Dámaso (1996). Teorías de la Traducción: Antología de textos. Cuenca: Ediciones de la Universidad de Castilla-La Mancha.

Ruiz Casanova, José Francisco (2000). Aproximación a una Historia de la Traducción en España. Cátedra: Madrid.

Sabio Pinilla, José Antonio (2009). La traducción en Portugal durante el siglo XVIII. En: José A. Sabio Pinilla (dir.), La traducción en la época ilustrada. Granada: Comares, 205-249.

Sabio Pinilla, José Antonio \& Ordóñez López, Pilar (2012). Las antologías sobre la traducción en el ámbito peninsular. Análisis y estudio. Berna: Peter Lang.

SAntoyo, Julio César (2009) La traducción medieval en la Península ibérica (siglos III-XV). León: Universidad de León.

Nolette, Nicole (2015): Jouer la traduction: théâtre et hétérolinguisme au Canada francophone. Ottawa: Les Presses de l'Université d'Ottawa, 285 p.

Dans cette œuvre phare qui «dresse le plan de la circulation en traduction de la production théâtrale de l'Ouest canadien francophone, de l'Ontario français et de l'Acadie» (p. 1), l'auteure élargit, sinon éclate, l'idée de la traduction au théâtre: il s'agit non pas d'une étude de la méthode, de l'histoire ou de la pratique de la traduction théâtrale au Canada, mais plutôt d'une analyse de la traduction comme actant, figurant et symbole qui inspirent, déterminent et soutiennent des pièces fortement ancrées dans un bilinguisme parfois ambigu, contesté et brouillé. Comme le suggère bien le titre, on ne joue pas en traduction ni avec la traduction: l'étude se livre à l'analyse des instances où il est question de «jouer la traduction» et se consacre à l'exploration de la traduction ludique dans le théâtre hétérolingue franco-canadien et du playsir du jeu entre les langues (p. 19). L'auteure précise que son étude porte sur la mise en scène de la traduction ludique dans ce théâtre, ainsi que sur la retraduction de ces spectacles dans un contexte de métropoles où le public ne maîtrise nécessairement qu'une des langues en jeu. On s'intéresse donc non pas à la manière dont la langue 